\title{
Differentiating physiology from pathology in elite athletes. Left ventricular hypertrophy versus hypertrophic cardiomyopathy
}

\author{
Hubert Krysztofiak ${ }^{1,2}$, Paweł Petkow Dimitrow ${ }^{3}$ \\ 'Mossakowski Medical Research Centre, Polish Academy of Sciences, Warsaw, Poland \\ ${ }^{2}$ National Centre for Sports Medicine, Warsaw, Poland \\ ${ }^{3} 2^{\text {nd }}$ Department of Cardiology, Collegium Medicum Jagiellonian University, Krakow, Poland
}

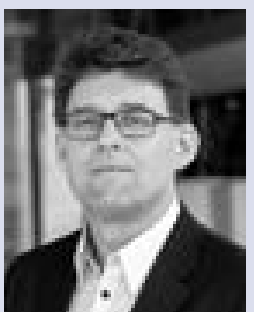

Hubert Krysztofiak, MD, PhD is the Head of the National Centre for Sports Medicine and an investigator at the Mossakowski Medical Research Centre of Polish Academy of Sciences. He is responsible for the medical care for Olympians. An important part of his activity is screening for, and management of, medical conditions that limit the ability of athletes to participate in sports training and competition or may predispose them to sudden cardiac death. He is the Chairman of the Medical Commission of the Polish Olympic Committee and the Chief Medical Officer for the Polish Olympic Team; a member of the Advisory Board of the IOC Diploma in Sports Medicine; and a member of the Sports Cardiology Section of the European Association for Cardiovascular Prevention and Rehabilitation (EACPR). He was the Chairman of the Working Group on Sports Cardiology of the Polish Cardiac Society and a Member of the Board of the Polish Society of Sports Medicine. His main area of scientific activity is cardiac adaptation to exercise in athletes and the clinical approach to cardiac pathology and problems in athletes.

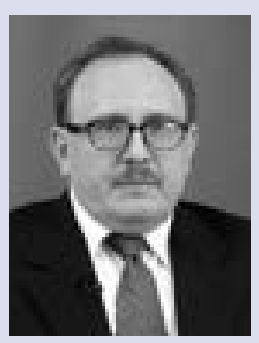

Professor Paweł Petkow Dimitrow, MD, PhD from the $2^{\text {nd }}$ Department of Cardiology at Collegium Medicum of Jagiellonian University. The main fields of his scientific interests include hypertrophic cardiomyopathy (HCM), storage diseases, and pathophysiology of coronary circulation. He is also interested in the topics of left ventricular hypertrophy and athlete's heart. He conducts research on biochemical biomarkers in HCM, and aortic stenosis and non-invasive assessment of the endothelial vasomotor function of coronary arteries. He introduced stress echocardiography in an upright position in the diagnostic examination of HCM patients. He is an author of original publications concerning HCM, which are recognised by the 2014 European Society of Cardiology Guidelines on diagnosis and management of HCM and the 2011 American Heart Association and American College of Cardiology Guidelines, as fundamental in research on HCM.

\section{INTRODUCTION}

When the heart is subjected to an additional haemodynamic load during a bout of exercise, it increases cross-bridge formation, according to the Frank-Starling law, and activates neurohormonal mechanisms to enhance contractility. However, the efficiency of these mechanisms during prolonged and repeated exercise is limited and it might be harmful as a chronic adaptation. Therefore, the heart attempts to enlarge its muscle to carry the extra load. In patients with hypertrophic cardiomyopathy (HCM), even a regular load is recognised as an excess haemodynamic burden, because of abnormal structural and functional changes in the sarcomeres. The impaired function of the sarcomeres is compensated by cardiac muscle enlargement [1,2]. Thus, the hypertrophy of cardiac muscle plays a key role in response to the absolute or relative, chronic increase of the haemodynamic load on the heart.

\section{Address for correspondence:}

Hubert Krysztofiak, MD, PhD, Mossakowski Medical Research Centre, Polish Academy of Sciences, ul. Pawińskiego 5, 02-106 Warszawa, Poland,

e-mail: hkrysztofiak@imdik.pan.pl

Received: 12.04.2016 Accepted: 13.04.2016 Available as AoP: 23.05.2016

Kardiologia Polska Copyright (C) Polskie Towarzystwo Kardiologiczne 2016 


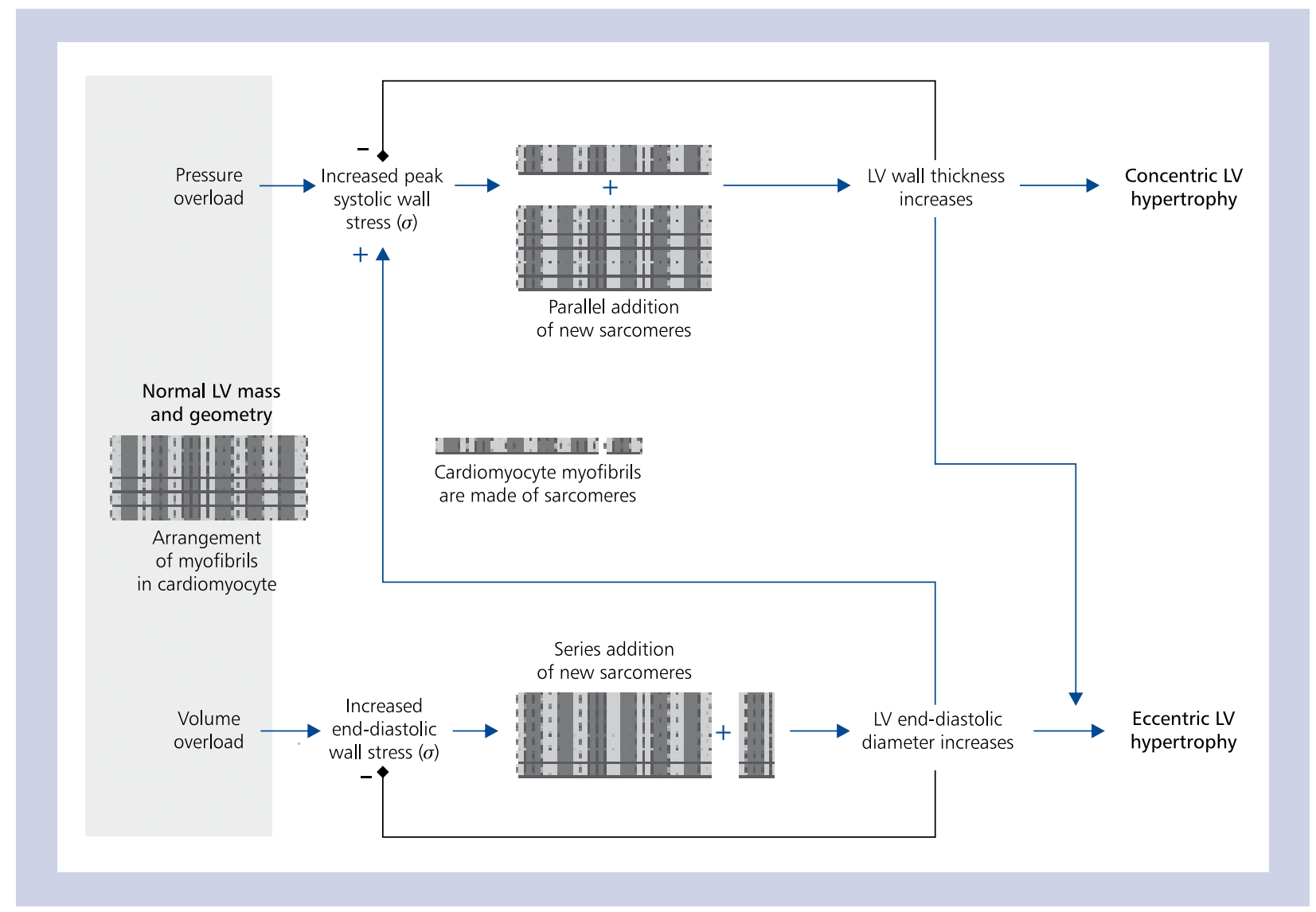

Figure 1. A drawing explaining Grossman's hypothesis about the patterns of hypertrophy related to wall stress. Volume overload induces an increase of end-diastolic wall stress. This is a stimulus for ventricular enlargement and an increase in the radius of the left ventricle (LV) in the mechanism of serial addition of new sarcomeres; the systolic wall stress increases. In response, a parallel addition of new sarcomeres gives balanced wall thickening and contributes to eccentric hypertrophy. Eventually, concentric hypertrophy causes a decrease in systolic wall stress in pressure overload, and eccentric hypertrophy reduces the diastolic wall stress in volume overload [3]

It is generally believed that mechanical signals initiate a cascade of biological events leading to cardiac growth. In 1975, William Grossman, Donald Jones, and Lambert P. McLaurin published a landmark work "Wall stress and patterns of hypertrophy in the human left ventricle" [3]. In their research, left ventricular hypertrophy (LVH) in patients with aortic stenosis was compared to $\mathrm{LVH}$, observed in patients with aortic and mitral regurgitation. The measurements of left ventricular (LV) dimensions and LV wall thickness (LVWT), recorded with one-dimensional echocardiography, and simultaneous measurements of pressure in the $\mathrm{LV}$, with high fidelity catheters, allowed matching the radius of the LV and LVWT and instantaneous pressure during the cardiac cycle.

In accordance with the law of Laplace, wall stress in the LV is proportional to the product of $L V$ pressure and radius and is inversely proportional to the thickness of the wall. Therefore, the pressure increase can be offset by an increase in the wall thickness. Because concentric hypertrophy develops under the influence of pressure overload and eccentric hypertrophy under the influence of volume overload, peak systolic wall stress has been proposed as a stimulus for parallel replication of the sarcomeres, causing concentric hypertrophy and end-diastolic wall stress, as a stimulus for serial replication of the sarcomeres and eccentric hypertrophy (Fig. 1).

The Working Group 'Myocardial Function' of the European Society of Cardiology (ESC), recommends using the term 'hypertrophy' only in the context of cardiac myocyte size, and not the whole heart [4]. Instead, the Working Group recommends using the term 'remodelling' to define the rearrangement of different cardiac tissue elements, a process by which the heart changes its size, geometry, and function over time. However, the terms remodelling and hypertrophy are often used interchangeably. In everyday clinical practice, an increase in the thickness of the LV wall beyond the limits established for a population is often regarded as hypertrophy. According to the guidelines published by Lang et al. [5], LVH is defined as a substantial increase in LV mass, expressed as LV mass, indexed to the body surface area of more than $95 \mathrm{~g} / \mathrm{m}^{2}$ for 


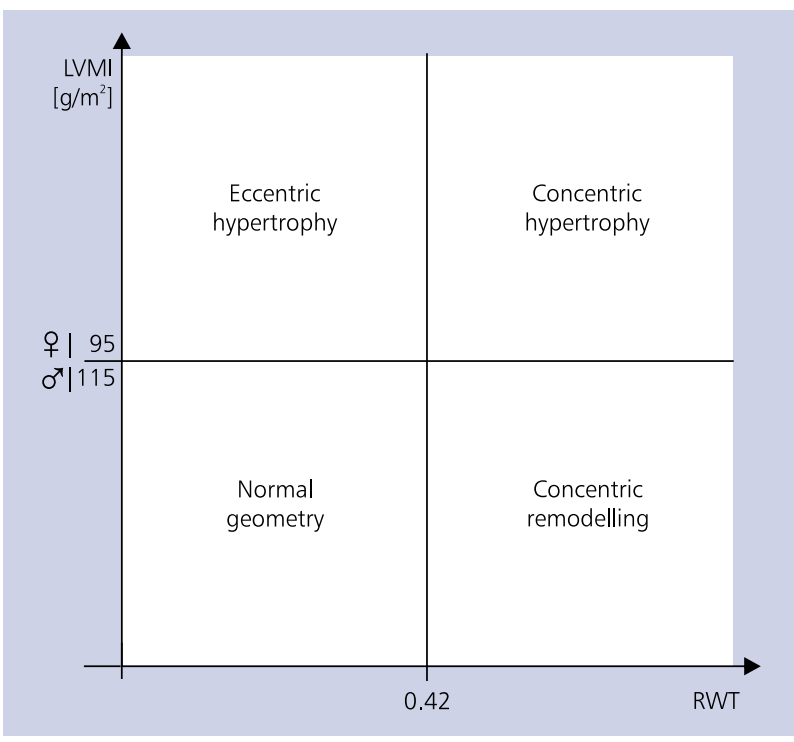

Figure 2. The four patterns of left ventricular geometry, proposed by Ganau et al. [6] in 1992 with modifications regarding LV mass index (LVMI) and relative wall thickness (RWT) introduced by Lang at al. [5]. Patients with normal LV mass can have either normal geometry if RWT $\leq 0.42$ or concentric remodelling if RWT $>0.42$. Patients with increased LV mass can have either concentric (RWT $>0.42)$ or eccentric (RWT $\leq 0.42)$ hypertrophy

female and $115 \mathrm{~g} / \mathrm{m}^{2}$ for male. Additionally, by calculating the relative wall thickness $(\mathrm{RWT})$, defined as $(2 \times \mathrm{PWT}) / \mathrm{LVEDD}$, where PWT is the posterior wall thickness and LVEDD is LV end-diastolic diameter, it is possible to perform a geometric distinction of the LV mass increase. If RWT is more than 0.42, hypertrophy is concentric; if RWT is equal to or less than 0.42 , the hypertrophy is eccentric (Fig. 2) [6].

\section{LEFT VENTRICULAR HYPERTROPHY OF ATHLETE'S HEART}

Hypertrophy of the LV in athletes represents cardiac remodelling in response to training. However, what is a stimulus for athlete's heart LV remodelling? I (HK) addressed this question to Bill Grossman, and he has compared the changes in athlete's heart to the natural growth of the heart with age, from the neonatal period to adulthood. He pointed out that volume overload during exercise generates both systolic and diastolic stress; therefore, the hypertrophy is balanced.

Fundamentally, LVH, as a feature of the athlete's heart, is regarded as balanced eccentric hypertrophy, in spite of the ongoing debate about the effect of resistance training on induction of concentric hypertrophy - a hypothesis introduced by Morganroth et al. in 1975 [7-9]. In 2000, Pluim et al. [8] presented a meta-analysis of 59 studies involving 1451 male athletes. The results confirmed the hypothesis of divergent cardiac adaptation in dynamic and static sports, respectively. This conclusion was based on a comparison of RWT in resistance trained athletes and endurance athletes. RWT in the resistance athletes was significantly higher than in the endurance and combined sports athlete groups. The mean values were estimated at 0.44, 0.39, and 0.40, respectively [8].

However, an analysis of the results of Pluim's research and the results of a recent meta-analysis by Utomi et al. [9] show that the adaptation is not necessarily divergent, but rather, the morphological features associated with specific training may represent consecutive points on a continuous line of cardiac growth, associated with exercise. If we assume the starting point of the line as the RWT of the control group, the course of RWT changes with training, regardless of the type of sport is directed toward the higher values. But, in healthy athletes, RWT should not reach the value defined as concentric hypertrophy (Table 1$)[8,9]$. It is interesting that, for RWT calculation, Pluim et al. [8] used the sum of interventricular septum (IVS) thickness and PWT and divided it by LVEDD, but Lang et al. [5], and also Ganau et al. [6], recommend the use of double PWT instead of the sum of IVS thickness and PWT. In this case, RWT is probably overestimated (Table 1).

Relative wall thickness is a function of LVWT and internal diameter. It is noteworthy that, in the meta-analysis of Pluim et al. [8], the differences between athlete groups were noted only for LVEDD and LV end-diastolic volume, which were larger in the endurance athletes than in the resistance athletes. There were no differences in the wall thickness between those groups. The same results were presented in the above-mentioned meta-analysis by Utomi et al. [9], in which the authors, contrary to the previous meta-analysis, concluded that the training-specific pattern of concentric hypertrophy was not discerned in the resistance athletes.

The strict classification of LV adaptation in the resistance athletes as concentric hypertrophy is probably not proper. The typical pattern of concentric hypertrophy is regarded as an adverse phenomenon. Even in hypertension one can observe eccentric hypertrophy or concentric remodelling, and concentric hypertrophy is a rare phenomenon, as was presented in the Ganau et al. work from 1992 [6].

The changes in size, geometry, and function of the heart are a result of the athlete's physical training and apply to almost all athletes. Of course, some sports disciplines require little training volume and intensity, and then the changes are just small. Nevertheless, unambiguously, athletes who are engaged in physical training have a greater LVWT and LVEDD, compared to the non-athletic population, and endurance athletes have the biggest hearts - those practicing rowing, cross-country skiing, cycling, and long-distance running. The aforementioned meta-analyses confirm this unequivocally $[8,9]$. Athletes also have proportionally larger left atria (LA) and right ventricles (RV), and this reflects a balanced cardiac remodelling process [9-12]. 
Table 1. The mean values of interventricular septum thickness (IVST), posterior wall thickness (PWT), and left ventricle end diastolic diameter (LVEDD) from the meta-analysis of Pluim et al. [8] and the meta-analysis of Utomi et al. [9] and the results of calculations of relative wall thickness with two methods

\begin{tabular}{|c|c|c|c|c|c|}
\hline \multirow[t]{2}{*}{ Athletes } & \multirow[t]{2}{*}{ PWT } & \multirow[t]{2}{*}{ IVST } & \multirow[t]{2}{*}{ LVEDD } & \multicolumn{2}{|c|}{ Relative wall thickness } \\
\hline & & & & $(2 \times$ PWT $) /$ LVEDD & $(\mathrm{PWT}+\mathrm{IVST}) / \mathrm{LVEDD}$ \\
\hline \multicolumn{6}{|c|}{ Pluim et al. 2000 [8] } \\
\hline Control & 8.8 & 8,8 & 49.6 & 0.35 & 0.35 \\
\hline Endurance & 10.3 & 10,5 & 53.7 & 0.38 & 0.39 \\
\hline Combined & 11 & 11.3 & 56.2 & 0.39 & 0.40 \\
\hline Resistance & 11 & 11.8 & 52.1 & 0.42 & 0.44 \\
\hline \multicolumn{6}{|c|}{ Utomi et al. 2013 [9] } \\
\hline Control & 8.8 & 9.2 & 50.1 & 0.35 & 0.36 \\
\hline Endurance & 10.6 & 11 & 54.8 & 0.39 & 0.39 \\
\hline Resistance & 10.4 & 11 & 52.4 & 0.40 & 0.41 \\
\hline
\end{tabular}

This adaptation process is very dynamic. First, changes on a molecular level can be observed at the beginning of the training regime. The changes are not uniform, and many determinants affect their course. However, an important feature of the athlete's heart is their reversibility after cessation of exercise, for example in detraining. The term "detraining" is commonly associated with athletes' activity; it describes withdrawal of morphological and physiological changes induced by training, after reduction of training intensity and volume, for example during the off-season or after injury. This phenomenon is considered as a feature distinguishing the athlete's heart from HCM.

Note that we usually think about the athlete's heart as a special entity, different from the normal reference ranges. However, what is the norm for us? These are our control groups. Groups of sedentary males and females. Did you ever think about it in an opposite way? To our knowledge, the human body evolved in an environment that required high physical activity. The human genome is programmed for high physical activity. Thus, it is possible that athletes should represent a reference point, and our control groups are in a permanent detraining period [13].

\section{THE ATHLETE'S HEART AND THE “GREY ZONE" OF UNCERTAINTY}

Sometimes, the magnitude of wall thickness or internal diameter of the LV resembles morphological manifestations of a pathological condition of the heart. Then, we have to remember that athletes are not a disease-free population. This is particularly important in the case of HCM because this disease is responsible for a substantial number of sudden cardiac deaths (SCD) in athletes [14]. The challenge starts when LVWT exceeds the lower limit of diagnostic value for $\mathrm{HCM}$ but is still below the upper limit observed in athletes.
According to the 2014 ESC guidelines on diagnosis and management of HCM, cardiomyopathies are defined by structural and functional abnormalities of ventricular myocardium that are unexplained by flow-limiting coronary artery disease or abnormal loading conditions. Specifically, HCM is defined by the presence of increased LVWT that is not solely explained by abnormal loading conditions [15].

In the section 'diagnostic criteria', the ESC guidelines state that in adults HCM is defined by LVWT equal or more than $15 \mathrm{~mm}$ in one or more myocardial segments, but also that the disorder can present with a lesser degree of wall thickening of $13 \mathrm{~mm}$ to $14 \mathrm{~mm}$. Therefore, according to the guidelines, all athletes with LVWT greater than $12 \mathrm{~mm}$ should be evaluated as suspected of HCM [15].

This is, more or less, consistent with recommendations of leading sports cardiologists. Only a small subset of white male athletes, estimated at 2-4\%, exceeds LVWT of $12 \mathrm{~mm}$ $[16,17]$. There is no evidence of LVWT greater than $16 \mathrm{~mm}$ in healthy male athletes regardless of ethnicity [18]. Therefore, the overlap is estimated at $12-13 \mathrm{~mm}$ to $16 \mathrm{~mm}$. In healthy female athletes, LVWT does not exceed $12 \mathrm{~mm}$. The female athletes have larger LV diameter and LVWT compared to sedentary females, but the extent of cardiac remodelling is less, compared to adult male athletes of the same ethnicity and sports discipline. This difference can be partly attributed to smaller body size, but it persists even after indexing to the body surface area. This is another feature considered as helpful in distinguishing the athlete's heart from HCM. Maximal LVWT greater than $12 \mathrm{~mm}$ in female athletes favours the diagnosis of HCM.

\section{DETERMINANTS OF CARDIAC SIZE IN ATHLETES}

Haemodynamic changes during intensive exercise are the stimulus for cardiac growth. However, a few factors affect the 
magnitude of cardiac remodelling in athletes. So far, we have referred to the type of sport and gender. The next important factor is the age of the athlete. LVWT and LV diameter increase in a linear manner from childhood to adulthood and correlate with changes in body size. Adolescent athletes have the greater LV diameter and LVWT compared to non-athletic, age-matched colleagues. However, the diameter and thickness are smaller than in adult athletes. Only a small subset exceeds $11 \mathrm{~mm}$ of LVWT. In the study by Sharma et al. [19] of 720 adolescent athletes it was $5 \%$, and only $0.4 \%$ exceeded $12 \mathrm{~mm}$. All female adolescent athletes had LVWT less than or equal to $11 \mathrm{~mm}$. The upper limit of the wall thickness in male adolescent athletes was $14 \mathrm{~mm}$. This difference, between the adolescent athletes and the adult athletes, should also be taken into account in the evaluation process, in the case of HCM suspicion.

Recently, particular attention has been paid to the impact of ethnicity on cardiac remodelling. Specifically, athletes of African or Afro-Caribbean origin exhibit significant differences compared with widely studied groups of Caucasian athletes. Black athletes have significantly larger LVWT and LV dimensions of the LA, but LVEDD is similar to that of the white athletes $[18,20]$.

In 2008, Basavarajaiah et al. [18] compared LV remodelling in highly-trained, male athletes of African and Afro-Caribbean ethnicity (black athletes) and the same level Caucasian athletes and showed, that the greater mean LV diameter and LVWT have black athletes. Additionally, they found that in black athletes there is an $18 \%$ greater prevalence of hypertrophy beyond the limit of $12 \mathrm{~mm}$, compared to white athletes. Although 3\% of black athletes had LVWT equal to or more than $15 \mathrm{~mm}$, none of the white and black athletes had LVWT more than $16 \mathrm{~mm}$.

Similar results were demonstrated in black female athletes in the study by Rawlins et al. [20]. LVH greater than $11 \mathrm{~mm}$ was found in $3 \%$ of black female athletes but in none of the white female athletes [20]. Thus, in the black athletes, adoption of the bottom LVWT limit of $12 \mathrm{~mm}$ will prompt us to HCM-oriented evaluation much more frequently, compared to the white athletes, and of course will generate a false positive diagnosis.

There is one more factor considered as a determinant of cardiac size in athletes: the genetic factor. Some authors suggest that the genetic determinants may explain one-quarter of the overall variability of LV dimensions. In particular, polymorphisms of the angiotensinogen gene (AGT) and the angiotensinogen converting enzyme (ACE) gene have been investigated, with the supposition that the AGT-TT genotype and the ACE-DD genotype are independently related to the greater LV mass after training, compared to the AGT-MM and ACE-II genotypes, respectively [21].

We are not making an error when we say that genes have an influence on heart size, since they have an impact on body size. It is not wrong even to say that genes independently have an influence on heart size. However, today our knowledge in this particular field is limited and the polymorphisms of renin-angiotensin system genes are a good example of these limitations. These genes are also considered to be determinants of HCM; so-called distant-acting modifiers. This phenomenon, independently of a direct genetic mutation, is in part responsible for the great phenotypic diversity observed in HCM, besides locally acting modifiers, epigenetic mechanisms, and environmental factors [22].

\section{HYPERTROPHIC CARDIOMYOPATHY}

Hypertrophic cardiomyopathy, defined by the presence of unexplained $\mathrm{LVH}$, affects 1 in 500 people, and the majority of the cases are caused by genetic mutations. It is the most common genetic heart disease $[15,23]$. The disease has heterogeneous morphology and variable clinical manifestations. The disease has complex pathophysiology, predominantly involving cardiomyocytes but also many different cells and tissues of the heart $[15,24]$.

Left ventricular hypertrophy is the phenotypic hallmark of HCM. It develops during adolescence or early adulthood, usually. However, HCM can be found at birth and can emerge at the senior age - so called late phenotypic presentation. Typical distribution of hypertrophy in the LV is asymmetric and regional [24]. It may involve one segment or virtually all walls of the LV, along with papillary muscles and the RV. A cardiac magnetic resonance imaging (CMR) study of Maron et al. [24] shows that in 54\% of HCM patients, hypertrophy is diffuse, involving more than seven segments of LV wall. In $34 \%$, hypertrophy is moderate - confined to three to seven segments. In the remaining $12 \%$ of HCM patients, only one or two segments are involved and this is regarded as focal hypertrophy. In $15 \%$ of patients, the hypertrophied segments might be separated by a wall of normal thickness [24].

It is noteworthy that focal or moderate distribution of hypertrophy with mild wall thickness is not necessarily associated with increased LV mass. The average hypertrophy, understood as increased wall thickness, confirmed in large cohort studies is estimated at $21-23 \mathrm{~mm}$, but in about $5-6 \%$ of cases a thickness of 13-15 $\mathrm{mm}$ can be found. On opposite side, extreme hypertrophy of $30 \mathrm{~mm}$ or more might be seen [24].

The first-line imaging modality applied in the evaluation of LVH is two-dimensional echocardiography. In athletes suspected of HCM all segments from the base to the apex should be precisely examined. The measurements should be done at the end-diastole and in short-axis views, at the level of the mitral valve, papillary muscles, and apex. Of course, all available views might be used to verify wall thickness, but it should be noted that the measurements in apical views might cause an overestimation. Special attention is needed regarding base segments of the IVS because of the possibility of inclusion of RV structures to measurements and risk of 
overestimation [25]. Right ventricular wall thickness should be evaluated too. The normal wall thickness of the RV is less than $5 \mathrm{~mm}$ in the parasternal long-axis view.

If any segments are not visualised properly to allow effective measurements, use of contrast agents should be considered $[25,15]$. In HCM patients, the most frequently hypertrophied regions of the LV are the anterior free wall and contiguous basal anterior septum. In these locations the wall thickness is commonly maximal. In the evaluation of $\mathrm{LVH}$, besides the absolute values of the measurements, estimation of the symmetry of the wall thickness distribution is important. According to the 2014 ESC guidelines, a septal-to-PWT ratio of 1.5 or more is suspicious [15]. We have to remember that in almost $50 \%$ of HCM patients LVH is confined to seven segments [24].

In about $20 \%$ of cases, echocardiography fails to give an adequate visualisation of LVH, especially in the lateral wall and apex. Therefore, CMR is recommended in HCM. It gives a clear separation of endocardial and epicardial borders and is considered the 'gold standard' to evaluate the wall thickness and diameter of the LV and RV [15]. Recently, apical HCM is recognised more frequently, due to better access to the CMR.

Left ventricular hypertrophy in HCM is often considered as concentric, and an LVEDD less than $45 \mathrm{~mm}$ favours the HCM diagnosis. Caselli et al. [26] compared a group of athletes with LVWT range 13-15 mm with HCM patients matched for LVWT. They concluded, that LV diameter represents the most reliable and independent criterion to differentiate physiologic from pathologic LVH. In their population, an LV diameter of $55 \mathrm{~mm}$ showed the highest sensitivity and specificity for distinguishing the athlete's heart from HCM. All the patients with HCM had LV diameter less than 55 mm [26]. In turn, Pagourelias et al. [27] in their study compared a group of healthy athletes to HCM patients engaged in regular exercise at least three times a week. In this study LVEDD, less than $48 \mathrm{~mm}$ (47.9) was estimated as the cut-off value, with sensitivity and specificity of $87.5 \%$ and $77.8 \%$, respectively. It is noteworthy that small LV size might be considered as a risk factor, predisposing to sudden death in HCM patients [28]. Additionally, small LV size at end-systole is characteristic for females with HCM. This configuration is frequently associated with significant obstruction of LV outflow tract (LVOT) [29].

Common features of HCM on the tissue level are cardiomyocyte disarray, microvascular dysfunction, and fibrosis [30]. The disarray is a profound disorganisation of cardiomyocyte alignment, expressed as a lack of parallel orientation of cells with additional branching intensifying an impression of disorder. Changes at the cellular level are associated with changes at the sarcomere level. The presence of disarray is independent of the degree of hypertrophy. A consequence of disarray is dysfunction of contraction and relaxation processes. It is also considered as a substrate for arrhythmias [30, 31].

Diffuse microvascular dysfunction in the heart, in the absence of changes in epicardial coronary arteries, is an ef- fect of pathological remodelling in small intramural vessels, with thickening of the arteriole wall, hyperplasia of smooth muscles, and disorganisation of elastic fibres [32]. A result of the remodelling process is a narrowing of the luminal diameter, most often irregular and impaired vasodilator reserve [33]. Therefore, microvascular dysfunction, along with compression forces, resulting from the hypertrophy may be a cause of ischaemia in HCM [32].

The additional and important consequence of microvascular dysfunction is fibrosis. Although earlier considered as a primary process in HCM patients, today it is believed that this is a reparative process, following microvascular dysfunction [34]. The process might range from increased, diffuse, interstitial collagen deposition to large focal areas of fibrosis, most often observed in mid-wall location, preferentially in the most hypertrophied regions.

In HCM patients, CMR with the gadolinium-based contrast agent, in late phase, may show an enhancement within the LV wall (the late gadolinium enhancement [LGE]), which corresponds to focal fibrosis [35]. The recently introduced method of measurement of myocardial longitudinal relaxation time (T1 mapping), following administration of gadolinium contrast agents, gives a chance to distinguish normal myocardium from diffuse myocardial fibrosis with standard CMR tools and machines [36]. LGE is present in about $60-90 \%$ of $\mathrm{HCM}$ patients, and the extent of LGE is inversely related to systolic function [37]. It is possible that LGE areas are also a substrate for ventricular arrhythmias [38].

The presence of LGE is generally considered as a confirmatory feature in case of suspected HCM. However, in patients with mild hypertrophy, LGE is frequently absent.

One of the defining features of HCM is LVOT obstruction. The obstruction is a result of morphological changes in the IVS, fibrous trigones and mitral valve [39]. During systole, a hypertrophied IVS comes in contact with an anterior leaflet of the mitral valve or even both elongated leaflets of the valve. This causes narrowing of the outflow tract and, consequently, blood flow acceleration and local under-pressure in the outflow tract (Venturi effect).

Several conditions must be met, but the most important are the changes in mitral valve apparatus resulting in a systolic anterior motion (SAM) of the anterior leaflet. In the majority of HCM patients both mitral leaflets are enlarged, which is a part of the HCM phenotype. The anterior papillary muscle is often displaced anteriorly and occasionally inserted directly into the mitral leaflet. It might be additionally complicated by fixation of papillary muscles to the ventricular free wall. The functional effect of the changes is mitral regurgitation, due to loss of the leaflets coaptation. Other elements are hypertrophy of LV wall below posterior mitral annulus and calcification of the posterior mitral annulus, causing a shift of the mitral valve leaflets anteriorly and the inability of the subaortic curtain to move backward during systole, due to the obliteration of 
fibrous trigones [39, 40]. M-mode echocardiography gives the opportunity to assess the severity of the systolic anterior motion. An incomplete SAM is recognised when, during cardiac cycle, the mitral valve leaflet does not touch IVS; mild, when a mitral-septal contact starts in late systole and lasts less than $10 \%$ of the systole; and severe, when the mitral-septal contact occurs in mid-systole and lasts more than $30 \%$ of the systole duration [41].

In advanced hypertrophy and particularly in elderly patients, LVOT obstruction is more commonly present with a deformation of hypertrophied septum, causing anatomic reduction of the subaortic area [40].

It should be noted, however, that in young people LVOT is often of normal size and arrangement, and the obstruction is caused by the wide excursion of elongated mitral leaflets. This type of obstruction is highly dynamic and varies in different physiological situations. Often not observed in resting conditions, it can be elicited during passive standing or exercise in an upright position, and sometimes during the Valsalva manoeuvre [42]. In this situation, drag forces deriving from high-velocity, abnormally directed anterograde systolic flow within the LV produce a systolic anterior motion. Increased flow velocity, secondary to the obstruction, quantified with continuous wave Doppler, has a characteristic dagger-shaped contour [43].

A gradient of $30 \mathrm{~mm} \mathrm{Hg}$ or more at rest or elicited with provocative manoeuvres defines the LVOT obstruction. Under resting conditions, the obstruction is found in about $30 \%$ of patients. In another $30-40 \%$, the obstruction is only seen after provocative manoeuvres [40, 43]. Therefore, exclusion of LVOT obstruction should be a priority of the clinical search, and the presence of SAM, even if incomplete, suggests the diagnosis.

In a significant subset of HCM patients with hypertrophied papillary muscles, an intraventricular gradient can be observed. In some of the patients, a mid-ventricular obstruction can be seen. Then, the gradient may be clinically relevant, and a consequence of the morphological and functional changes can be the occurrence of the LV apical aneurysm, which is observed in about $2 \%$ of HCM patients [44].

Another feature of HCM is LA remodelling. It is probably due to LV diastolic dysfunction and increased filling pressure [45]. An additional factor can be mitral regurgitation resulting from SAM. There is also a hypothesis that the atrial myopathy is a primary pathology; an effect of an HCM-causing mutation. A transverse dimension of the LA exceeds the reference value of $40 \mathrm{~mm}$ in more than half patients with $\mathrm{HCM}$, compared to $20 \%$ of athletes. In only $2 \%$ of athletes the LA dimension is equal to or more than $45 \mathrm{~mm}$, comparing to more than $25 \%$ of HCM patients [10]. Therefore, the LA dimension of more than $45 \mathrm{~mm}$ may favour the diagnosis of HCM. However, in the aforementioned study by Caselli et al. [26], in HCM patients with mild hypertrophy, LA size was within normal limits and smaller than in athletes.
One more pathology in HCM, responsible for ischaemia, is myocardial bridging, and sometimes even myocardial tunnelling. In patients with HCM the myocardial bridging occurs more frequently than in the general population; the prevalence rate is about $30 \%$, compared to $1 \%$ to $3 \%$, respectively. Myocardial bridging occurs when epicardial coronary arteries are located intramyocardially, resulting in systolic compression of a coronary artery [46].

\section{THE HYPERTROPHIC CARDIOMYOPATHY AETIOLOGY - GENETICS AND BEYOND}

As mentioned earlier, the majority of HCM cases are caused by genetic mutations. Among these, most common are mutations that affect sarcomere proteins [22]. In 1990, a mutation responsible for HCM was identified in the beta-myosin heavy chain (MYH7) gene. During the following decade, numerous genes were reported to be associated with the disease. Most of these genes encode proteins of myofilaments or the Z-disc of the sarcomeres. As such, HCM has been described as 'a disease of the sarcomere', and this aetiology is most often considered in a case of $\mathrm{LVH}$ that is not explained by increased loading conditions [22].

Among sarcomere genes, which clearly proved to have a pathogenic role in $\mathrm{HCM}$, most often recognised are the cardiac myosin-binding protein C (MYBPC3) gene, a gene encoding the MYH7 of thick filaments of the sarcomeres, and the cardiac troponin T (TNNT2) and I (TNNI3) genes of thin filaments, respectively. Rare variants have been reported in other genes of the sarcomere apparatus, or genes coding for proteins of the adjacent Z-disc. However, for some of these latter genes, evidence for direct pathogenicity in HCM has not been clearly established [22].

Over the past two decades, more than 1400 mutations have been described in association with HCM. The majority of them are familial mutations, but $40 \%$ are apparently sporadic cases with no family history. Almost all mutations associated with HCM are 'private mutations', identified in one or only a few families. However, some mutations arise recurrently in a large number of unrelated families because of mutations in mutational 'hot spots' - fragile regions of DNA. Other interesting cases are founder mutations: very old mutations persisting in a population because of their benign course and minimal impact on survival. Some of them may be older than thirty thousand years. The founder mutations almost universally occur in the MYBPC3 [47].

Pathogenic mutations are often missense substitutions, resulting in an exchange of a single amino acid for another at the protein level. Alternatively, and particularly in the MYBPC3, structural changes or truncation of the protein are identified. The final result is disruption of normal sarcomere function or haploinsufficiency [47].

What is the impulse for remodelling in HCM? Altered calcium handling is universally reported in sarcomeric car- 
diomyopathies and is considered as a probable primary or secondary trigger for pathological hypertrophy and some other features of HCM [2]. Sarcomeric mutations may cause sustained elevation of intracellular calcium by two mechanisms: increased sarcomeric adenosine triphosphate (ATP) utilisation, which may lead to down-regulation of sarcoplasmic reticulum ATPase and, consequently, impaired calcium removal, and increased myofilament calcium sensitivity, which slows its dissociation from the myofilaments and contributes to increased concentration during diastole. Sustained elevation of calcium concentration leads to activation of calcineurin phosphatase. The calcineurin then dephosphorylates a nuclear factor of activated T-cells (NFAT). The transcription factor, after translocation to the nucleus, induces genes responsible for pathological cardiac hypertrophy [48].

On the other hand, experimental studies have shown that one of the beneficial effects of regular exercise might be a down-regulation of the calcineurin signalling, competing with the pathological up-regulation observed in HCM. Thus, taking into account the lack of comparative data regarding the effect of exercise on HCM patients, it is worth considering, whether exclusion from exercise and sport for every asymptomatic HCM patient is the best way to save a life [48].

\section{THE CHALLENGE OF DIFFERENTIAL DIAGNOSIS}

Two overlapping circles symbolise the challenge of differentiating the athlete's heart from HCM. As was mentioned above, in a subset of athletes the maximum LVWT overlaps with that observed in HCM patients. In about $5-6 \%$ of the patients the maximum wall thickness is below the diagnostic value of $15 \mathrm{~mm}$ and may be of 13-14 $\mathrm{mm}$. In turn, about $4 \%$ of white athletes and even more black athletes have wall thickness greater than $12 \mathrm{~mm}$. Therefore, we must assume that every athlete whose LVWT is beyond $12 \mathrm{~mm}$ needs special attention and exclusion of HCM.

It is generally recommended to withdraw an athlete with "probable or unequivocal clinical diagnosis of hypertrophic cardiomyopathy" from most competitive sports because of increased risk of SCD, which is associated with vigorous exercise [51, 52]. However, it is important to recognise that in the case of HCM there is still a deficit of randomised and controlled clinical trials, and the recommendations are based on expert opinions. We have to accept that the established approach in differential diagnosis promotes sensitivity at the expense of specificity, and we have to face the problem of an apparently healthy athlete excluded from the sport because the athlete's safety is the first priority.

A few features recognised as important in the differentiation process are listed in Table 2. The presence of the features favours the diagnosis of HCM. Some of them result from medical history and physical examination. Some of them we can find in the resting electrocardiogram (ECG) because electrical remodelling is a part of both entities [53]. Some of them are
Table 2. Features recognised as important in differentiation of left ventricular hypertrophy (LVH) related to exercise from hypertrophic cardiomyopathy (HCM)

Specific data from personal and family history relevant to diagnosis of HCM (detailed in Table 3)

Cardiovascular findings in physical examination

Heart murmur

Electrocardiogram abnormal findings

T-wave inversion; ST depression; pathological Q;

LBBB; Intraventricular conduction delay;

Left axis deviation; LA enlargement;

RVH pattern; PVCs; ventricular arrhythmias

Cardiac structural features

LV wall thickness $>16 \mathrm{~mm}$ in adult male and $>12 \mathrm{~mm}$ in adult female athlete regardless of ethnicity

$\mathrm{LV}$ wall thickness $>14 \mathrm{~mm}$ in adolescent male and $>11 \mathrm{~mm}$ in adolescent female athlete

Asymmetrical or unusual LVH (IVST/PWT ratio $\geq 1.5$ );

LVEDD < 45 mm; LA > 45 mm; RVH;

LVOTO; SAM;

Late gadolinium enhancement on CMR;

Myocardial bridging

Functional abnormalities

E wave $<$ A wave;

TDI e' wave $<9 \mathrm{~cm} / \mathrm{s}$;

Ar-A duration > 30 ms;

Increased BNP;

Low $\mathrm{VO}_{2} \max$

No effect of detraining to $\mathrm{LVH}$

Genetics

A wave - pulsed-wave mitral peak velocity of late filling; BNP B-type natriuretic peptide; CMR — cardiac magnetic resonance imaging; E wave - pulsed-wave mitral peak velocity of early filling; IVST — intraventricular septum thickness; LA — left atrium; LBBB - left bundle branch block; LVEDD — left ventricular end-diastolic diameter; LVOTO - left ventricular outflow tract obstruction; PWT — posterior wall thickness; PVCs — premature ventricular contractions; RVH right ventricle hypertrophy; SAM — systolic anterior motion; TDI $\mathrm{e}^{\prime}$ wave - tissue Doppler imaging early diastolic peak velocity; $\mathrm{VO}_{2} \mathrm{max}$ - maximal oxygen uptake

the result of imaging diagnostics - from the evaluation of structure and geometry to advanced functional analysis. Others, like reduction of the wall thickness after detraining and the level of maximal oxygen uptake, are based on responses to prolonged immobilisation and exercise, respectively. One biochemical parameter is listed in this table: the B-type natriuretic peptide, recognised as a marker of heart failure. Finally, there is genetic analysis, which is highly specific and is becoming more available, but demands new competencies and skills for the conclusive application.

Separately, we would like to discuss the functional abnormalities, with a focus on LV diastolic dysfunction, as an early 
symptom of HCM and the family history, the procedure which as the rule should start every clinical investigation. Here, in the context of the sarcomere mutations.

\section{LEFT VENTRICULAR DIASTOLIC FUNCTION IN HYPERTROPHIC CARDIOMYOPATHY}

During the normal cardiac cycle, just after closure of the aortic valve, the stage of diastole starts. It begins with an isovolumic ventricular relaxation. This active process initiates a fall of pressure in the LV. When the LV pressure is equal to the LA pressure, the mitral valve opens and rapid inflow to the LV starts. At this point, the isovolumic relaxation ends, but myocardial relaxation continues during early diastole until the minimal diastolic pressure in the LV is reached. The blood is sucked to the LV. Rapid inflow causes the LV pressure to rise and equalise with the pressure in the LA, and thus early diastolic filling decelerates. Then the phase of diastasis starts. The filling of the LV almost closes the mitral valve leaflets, and then atrial contraction starts. The pressure in the LA rises and this initiates the late filling. Once again the mitral annulus moves away from the apex. During LA contraction, a small portion of blood goes back to the pulmonary veins. The mitral valve closes and this is the end of diastole.

Impairment of LV diastolic function may be an early sign of HCM development, and it is a consequence of disturbances in calcium handling, because of sarcomeric mutation. Diastolic function is related to the active process of relaxation and passive properties of the myocardium. However, the relaxation is impaired, at first, causing an increase of minimum LV end-diastolic pressure and consequently a decrease of early diastolic velocity of the mitral inflow and prolongation of the deceleration time [54]. In this situation, the conduit LA volume decreases, and the pump function gains importance. The late diastolic velocity of the mitral inflow increases. An increase in the LV filling pressure generates an increase of the LA pressure, and during atrial contraction a retrograde flow to the pulmonary veins, observed as the atrial reversal velocity, is magnified. Relaxation is accompanied by the movement of the mitral annulus away from the apex. Its velocity correlates well with how fast the LV relaxes.

Doppler parameters of diastolic function in athletes and the untrained controls were analysed in the work of Caselli et al. [55]. A significantly lower mitral inflow pulsed-wave late diastolic filling (A-wave) peak velocity in the athletes group confirms that in athletes the reservoir and conduit function of the LA predominates. The same effect was observed in the study by D'Ascenzi et al. [56] of 150 athletes compared to 90 matched controls.

Some doubts might arise regarding the seemingly unfavourable direction of change of the isovolumic relaxation time and deceleration time, but bradycardia and physiological hypertrophy are mainly responsible for this picture.
In the athlete's heart the majority of the LV filling occurs in early diastole, because of effective suction forces generated by relaxation. The blood flow meets almost no resistance because of good compliance, and the LV filling pressure is low even during strenuous exercise. Generally, the diastolic reserve is large and all the processes of active relaxation, passive filling, and LA contraction are very well organised [55, 56].

Non-invasive estimation of diastolic function in HCM patients, especially in those with preserved or even enhanced systolic function, is difficult [57]. It is even more difficult in athletes with HCM because there is a lack of comparative studies of athletes with HCM and healthy athletes. Some experts believe that echocardiographic assessment of diastolic function in HCM has no clinical value [43]. However, if we assume that in healthy athletes the diastolic stage is a very well designed process then it is worth analysing some variables. The most recommended is the tissue Doppler annular early diastolic velocity. A value less than $9 \mathrm{~cm} / \mathrm{s}$ is considered as favouring the diagnosis of $\operatorname{HCM}[15,57]$.

From the perspective of diastolic function, reduced tissue Doppler annular early diastolic velocity is consistent with HCM. Prominent atrial reversal velocity in the pulmonary veins is also consistent with pathologic LVH and could also be of value $[25,57]$.

In everyday clinical practice the atrial reversal velocity and its derivatives are often treated as old variables and are rarely performed. However, they have a significant correlation with LV diastolic pressure and the time difference between atrial reversal velocity duration and mitral inflow A-wave duration; more than $30 \mathrm{~ms}$ indicates elevated LV end-diastolic pressure [25].

\section{FAMILY HISTORY OF HCM}

In 1958 Donald Teare described cases of young adults who died suddenly [58]. An autopsy revealed asymmetric LVH. In 1960 Hollman et al. [59] published the family pedigree of one of the patients, a 21-year-old woman. Besides family history and a review of medical records, including three autopsy reports, a clinical evaluation in living family members was performed. The result of the analysis revealed for the first time the hereditary nature of the disease. They described this entity as a 'dominant one, not sex-linked'. It means that every child of an HCM patient has a 50\% chance of being affected. It means that parents and every sibling of the patient have a very high probability of being affected.

Hollman's analysis shows how effective this kind of inquiry can be. Today, with easy access to sophisticated imaging techniques, we sometimes forget to put special emphasis on such 'basic' medical examination procedures, such as family history, limiting inquiry into questions about the medical history of first-degree relatives. However, in HCM, which is predominantly a result of autosomal dominant mutation, com- 
Table 3. Specific data from personal and family history relevant to diagnosis of hypertrophic cardiomyopathy

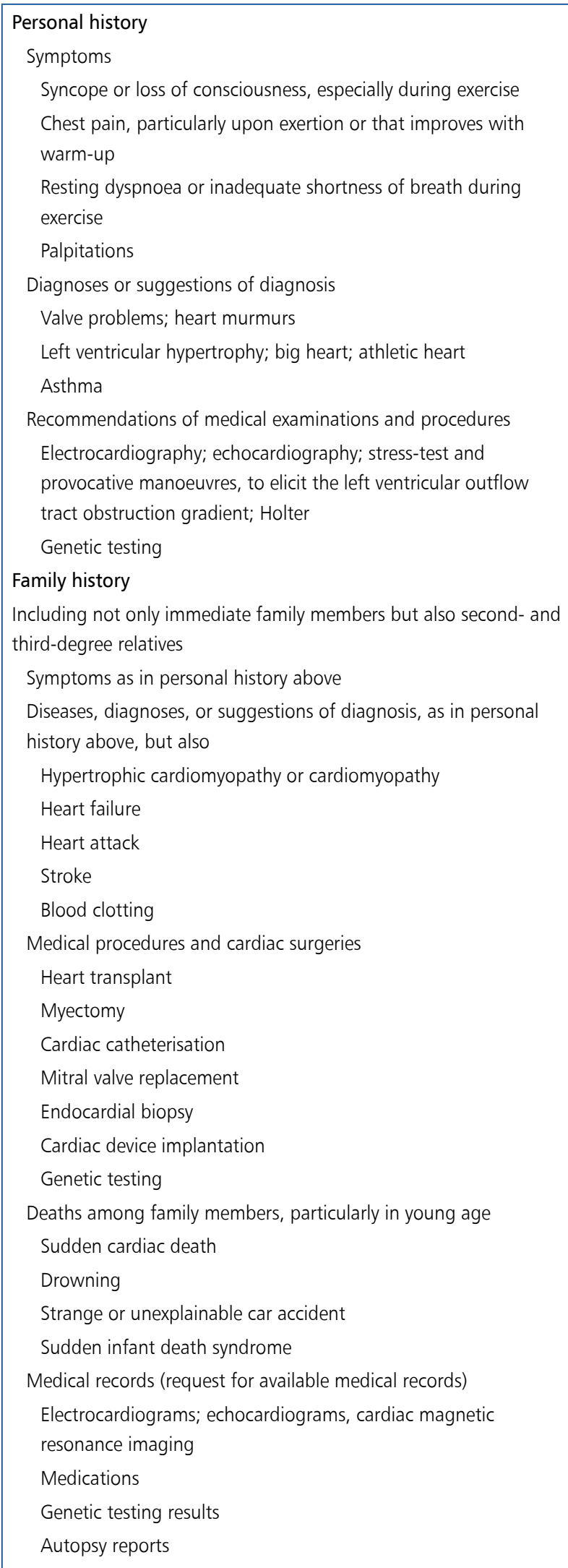

prehensive, multi-generational family history complemented by a review of available medical records and followed by clinical evaluation of the athlete's immediate family members, may be of great value.

In the case of an athlete with LVH that raises any doubts, the first step in the clinical investigation should be a thorough, three-generation family history. The occurrence of SCD among family members is crucial information [60]. However, we should also ask about heart failure and stroke, cardiothoracic surgeries, and others procedures, particularly in young age, which may have been a result of HCM. We need information on the occurrence of typical symptoms of HCM, like shortness of breath, chest pain, palpitations, and syncope, particularly associated with exertion, especially in younger family members. It is worth asking about cardiac diagnoses, using customary, simple terms. Do not forget about other related events, such as drowning, or a strange or unexplainable car accident in which a family member was the driver, or sudden infant death - these may suggest sudden cardiac arrest (Table 3).

The consequence of the inquiry should be an analysis of available medical records of family members, particularly ECGs and echocardiograms. The next step should be a clinical evaluation of selected members of immediate family, with ECG and echocardiography and other tests, depending on clinical context.

The great phenotypic diversity of HCM, even within the same family, means that unequivocally distinguishing the disease from the athlete's heart is often difficult. Therefore, differentiation is possible using a methodical and comprehensive clinical approach.

However, we have to remember that we do not know the cumulative effect of regular exercise and sarcomeric mutation on hypertrophy development in athletes, and we still do not know what the interrelations between regular exercise and HCM are. A lack of direct comparative studies of athletes with physiological and pathological hypertrophy limits the accuracy of recommended criteria.

Sarcomeric HCM is often an asymptomatic, benign disease with normal life expectancy. Advanced methods of genetic testing, although with ethical limitations, can give a chance for effective diagnosis in the near future. However, some identified mutations probably have no clinical relevance and introduce confusion into the accurate interpretation of genetic testing.

\section{Conflict of interest: none declared}

\section{References}

1. Georgakopoulos D, Christe ME, Giewat M et al. The pathogenesis of familial hypertrophic cardiomyopathy: early and evolving effects from an alpha-cardiac myosin heavy chain missense mutation. Nat Med, 1999; 5: 327-330. doi: 10.1038/6549.

2. Somura F, Izawa $\mathrm{H}$, Iwase $\mathrm{M}$ et al. Reduced myocardial sarcoplasmic reticulum $\mathrm{Ca}(2+)$-ATPase mRNA expression and biphasic force-frequency relations in patients with hyper- 
trophic cardiomyopathy. Circulation, 2001; 104: 658-663. doi: 10.1161/hc3101.093869.

3. Grossman W, Jones D, McLaurin LP. Wall stress and patterns of hypertrophy in the human left ventricle. J Clin Invest, 1975; 56: 56-64. doi: 10.1172/JCI108079.

4. Knöll R, Iaccarino G, Tarone $\mathrm{G}$ et al. Towards a re-definition of "cardiac hypertrophy" through a rational characterization of left ventricular phenotypes: a position paper of the Working Group "Myocardial Function" of the ESC. Eur J Heart Fail, 2011; 13: 811-829. doi: 10.1093/eurjhf/hfr071.

5. Lang RM, Badano LP, Mor-Avi V et al. Recommendations for Cardiac Chamber Quantification by Echocardiography in Adults: An Update from the American Society of Echocardiography and the European Association of Cardiovascular Imaging. Eur Heart J Cardiovasc Imaging, 2015; 16: 233-271. doi:10.1093/ehjci/jev014.

6. Ganau A, Devereux RB, Roman MJ et al. Patterns of left ventricular hypertrophy and geometric remodeling in essential hypertension. J Am Coll Cardiol, 1992; 19: 1550-1558. doi: 10.1016/0735-1097(92)90617-V.

7. Morganroth J, Maron BJ, Henry WL, Epstein SE. Comparative left ventricular dimensions in trained athletes. Ann Intern Med, 1975; 82: 521-524. doi:10.7326/0003-4819-82-4-521.

8. Pluim BM, Zwinderman AH, van der Laarse A, van der Wall EE. The Athlete s Heart: A Meta-Analysis of Cardiac Structure and Function. Circulation, 2000; 101: 336-344. doi: 10.1161/01. CIR.101.3.336.

9. Utomi V, Oxborough D, Whyte GP et al. Systematic review and meta-analysis of training mode, imaging modality and body size influences on the morphology and function of the male athlete's heart. Heart, 2013; 99: 1727-1733. doi: 10.1136/heartjnl-2012-303465.

10. Pelliccia A, Maron BJ, Di Paolo FM et al. Prevalence and clinical significance of left atrial remodeling in competitive athletes. J Am Coll Cardiol, 2005; 46: 690-696. doi: 10.1016/j.jacc.2005.04.052.

11. D’Ascenzi F, Pelliccia A, Corrado D et al. Right ventricular remodelling induced by exercise training in competitive athletes. Eur Hear J Cardiovasc Imaging, 2015; 17: 301-307. doi: 10.1093/ehjci/jev155.

12. Król W, Braksator W, Kasprzak JD et al. The influence of extreme mixed exertion load on the right ventricular dimensions and function in elite athletes: a tissue Doppler study. Echocardiography, 2011; 28: 753-760. doi: 10.1111/j.1540-8175.2011.01437.x.

13. Booth FW, Chakravarthy M V, Spangenburg EE. Exercise and gene expression: physiological regulation of the human genome through physical activity. J Physiol, 2002; 543: 399-411. doi: 10.1113/jphysiol.2002.019265.

14. Maron BJ, Doerer JJ, Haas TS et al. Sudden deaths in young competitive athletes: analysis of 1866 deaths in the United States, 1980-2006. Circulation, 2009; 119: 1085-1092. doi: 10.1161/CIRCULATIONAHA.108.804617.

15. Elliott PM, Anastasakis A, Borger MA et al. 2014 ESC Guidelines on diagnosis and management of hypertrophic cardiomyopathy: The Task Force for the Diagnosis and Management of Hypertrophic Cardiomyopathy of the European Society of Cardiology (ESC). Eur Heart J, 2014; 35: 2733-2779. doi: 10.1093/eurheartj/ehu284.

16. Pelliccia A, Maron BJ, Spataro A et al. The Upper Limit of Physiologic Cardiac Hypertrophy in Highly Trained Elite Athletes. N Engl JMed, 1991; 324: 295-301. doi: 10.1056/NEJM199101313240504.

17. Pelliccia A, Maron BJ, Culasso F et al. Athlete's heart in women: Echocardiographic characterization of highly trained elite female athletes. JAMA, 1996; 276: 211-215. doi: 10.1001/jama.1996.03540030045030.

18. Basavarajaiah $\mathrm{S}$, Boraita $\mathrm{A}$, Whyte $\mathrm{G}$ et al. Ethnic differences in left ventricular remodeling in highly-trained athletes relevance to differentiating physiologic left ventricular hypertrophy from hypertrophic cardiomyopathy. J Am Coll Cardiol, 2008; 51: 2256-2262. doi: 10.1016/j.jacc.2007.12.061.
19. Sharma S, Maron BJ, Whyte G et al. Physiologic limits of left ventricular hypertrophy in elite junior athletes: relevance to differential diagnosis of athlete's heart and hypertrophic cardiomyopathy. J Am Coll Cardiol, 2002; 40: 1431-1436. doi: 10.1016/S0735-1097(02)02270-2.

20. Rawlins J, Carre F, Kervio G et al. Ethnic differences in physiological cardiac adaptation to intense physical exercise in highly trained female athletes. Circulation, 2010; 121: 1078-1085. doi: 10.1161/CIRCULATIONAHA.109.917211.

21. Luo R, Li X, Wang Y et al. The Influence of Angiotensin Converting Enzyme and Angiotensinogen Gene Polymorphisms on Hypertrophic Cardiomyopathy. PLoS One, 2013; 8: e77030. doi:10.1371/journal.pone.0077030.

22. Ho CY, Charron P, Richard P et al. Genetic advances in sarcomeric cardiomyopathies: state of the art. Cardiovasc Res, 2015; 105 : 397-408. doi: 10.1093/cvr/cvv025.

23. Maron BJ, Gardin JM, Flack JM et al. Prevalence of hypertrophic cardiomyopathy in a general population of young adults. Echocardiographic analysis of 4111 subjects in the CARDIA Study. Circulation, 1995; 92: 785-789. doi: 10.1161/01.CIR.92.4.785.

24. Maron MS, Maron BJ, Harrigan C et al. Hypertrophic cardiomyopathy phenotype revisited after 50 years with cardiovascular magnetic resonance. J Am Coll Cardiol, 2009; 54: 220-228. doi: 10.1016/j.jacc.2009.05.006.

25. Nagueh SF, Bierig SM, Budoff MJ et al. American Society of Echocardiography clinical recommendations for multimodality cardiovascular imaging of patients with hypertrophic cardiomyopathy: endorsed by the American Society of Nuclear Cardiology, Society for Cardiovascular Magnetic Resonance, and Society of Cardiovascular Computed Tomography. J Am Soc Echocardiogr, 2011; 24: 473-498. doi: 10.1016/j.echo.2011.03.006.

26. Caselli S, Maron MS, Urbano-Moral JA et al. Differentiating left ventricular hypertrophy in athletes from that in patients with hypertrophic cardiomyopathy. Am J Cardiol, 2014; 114: 1383-1389. doi: 10.1016/j.amjcard.2014.07.070.

27. Pagourelias ED, Efthimiadis GK, Kouidi E et al. Efficacy of various "classic" echocardiographic and laboratory indices in distinguishing the "gray zone" between athlete's heart and hypertrophic cardiomyopathy: a pilot study. Echocardiography, 2013; 30: 131-139. doi: 10.1111/echo.12014.

28. Dimitrow PP, Dubiel JS. Echocardiographic risk factors predisposing to sudden cardiac death in hypertrophic cardiomyopathy. Heart, 2005; 91: 93-94. doi: 10.1136/hrt.2003.030353.

29. Dimitrow PP, Czarnecka D, Kawecka-Jaszcz K, Dubiel JS. The influence of age on gender-specific differences in the left ventricular cavity size and contractility in patients with hypertrophic cardiomyopathy. Int J Cardiol, 2003; 88: 11-16. doi: 10.1016/S0167-5273(02)00323-6.

30. Olivotto I, Giromami F, Nistri S et al. The many faces of hypertrophic cardiomyopathy: from developmental biology to clinical practice. J Cardiovasc Transl Res, 2009; 2:349-367. doi: 10.1007/s12265-009-9137-2.

31. McCauley MD, Wehrens XHT. Animal models of arrhythmogenic cardiomyopathy. Dis Model Mech, 2009; 2: 563-570. doi: 10.1242/dmm.002840.

32. Cecchi F, Sgalambro A, Baldi M et al. Microvascular dysfunction, myocardial ischemia, and progression to heart failure in patients with hypertrophic cardiomyopathy. J Cardiovasc Transl Res, 2009; 2: 452-461. doi: 10.1007/s12265-009-9142-5.

33. Dimitrow PP, Krzanowski M, Nizankowski R et al. Comparison of the effect of verapamil and propranolol on response of coronary vasomotion to cold pressor test in symptomatic patients with hypertrophic cardiomyopathy. Cardiovasc Drugs Ther, 2000; 14: 643-650.

34. Ho CY, Lopez B, Coelho-Filho OP et al. Myocardial fibrosis as an early manifestation of hypertrophic cardiomyopathy. N Engl J Med, 2010; 363: 552-563. doi: 10.1056/NEJMoa1002659. 
35. Maron MS, Appelbaum E, Harrigan CJ et al. Clinical profile and significance of delayed enhancement in hypertrophic cardiomyopathy. Circ Heart Fail, 2008; 1: 184-191. doi: 10.1161/CIRCHEARTFAILURE.108.768119.

36. Kellman P, Hansen MS. T1-mapping in the heart: accuracy and precision. J Cardiovasc Magn Reson, 2014; 16: 2. doi: 10.1186/1532-429X-16-2.

37. Nagueh SF, Bachinski LL, Meyer D et al. Tissue Doppler imaging consistently detects myocardial abnormalities in patients with hypertrophic cardiomyopathy and provides a novel means for an early diagnosis before and independently of hypertrophy. Circulation, 2001; 104: 128-130. doi: 10.1161/01.CIR.104.2.128.

38. Dimitrow PP, Klimeczek P, Vliegenthart $\mathrm{R}$ et al. Late hyperenhancement in gadolinium-enhanced magnetic resonance imaging: comparison of hypertrophic cardiomyopathy patients with and without nonsustained ventricular tachycardia. Int J Cardiovasc Imaging, 2008; 24: 77-83.

39. Klues HG, Maron BJ, Dollar AL, Roberts WC. Diversity of structural mitral valve alterations in hypertrophic cardiomyopathy. Circulation, 1992; 85: 1651-1660. doi: 10.1161/01.CIR.85.5.1651.

40. Lewis JF, Maron BJ. Elderly patients with hypertrophic cardiomyopathy: a subset with distinctive left ventricular morphology and progressive clinical course late in life. J Am Coll Cardiol, 1989; 13: 36-45. doi:10.1016/0735-1097(89)90545-7.

41. Gilbert BW, Pollick C, Adelman AG, Wigle ED. Hypertrophic cardiomyopathy: Subclassification by M mode echocardiography. Am J Cardiol, 1980; 45: 861-872. doi: 10.1016/00029149(80)90133-2.

42. Dimitrow PP, Bober M, Michałowska J, Sorysz D. Left ventricular outflow tract gradient provoked by upright position or exercise in treated patients with hypertrophic cardiomyopathy without obstruction at rest. Echocardiography, 2009; 26: 513-520. doi: wiley.com/10.1111/j.1540-8175.2008.00851.x.

43. Pandian NG, Rowin EJ, Gonzalez AMG, Maron MS. Echocardiographic profiles in hypertrophic cardiomyopathy: imaging beyond the septum and systolic anterior motion. Echo Res Pract, 2015; 2: E1-E7. doi: 10.1530/ERP-15-0009.

44. Cecchi F, Olivotto I, Nistri S et al. Midventricular obstruction and clinical decision-making in obstructive hypertrophic cardiomyopathy. Herz, 2006; 31: 871-876. doi: 10.1007/s00059006-2928-1.

45. Nistri S, Olivotto I, Betocchi S et al. Prognostic significance of left atrial size in patients with hypertrophic cardiomyopathy (from the Italian Registry for Hypertrophic Cardiomyopathy). Am J Cardiol, 2006; 98: 960-965. doi: 10.1016/j.amjcard.2006.05.013.

46. Sorajja P, Ommen SR, Nishimura RA et al. Myocardial bridging in adult patients with hypertrophic cardiomyopathy. J Am Coll Cardiol, 2003; 42: 889-894. doi:10.1016/S0735-1097(03)00854-4.

47. Seidman CE, Seidman JG. Identifying sarcomere gene mutations in hypertrophic cardiomyopathy: A personal history. Circ Res, 2011; 108: 743-750. doi: 10.1161/CIRCRESAHA.110.223834.
48. Konhilas JP, Watson PA, Maass A et al. Exercise can prevent and reverse the severity of hypertrophic cardiomyopathy. Circ Res, 2006; 98: 540-548. doi: 10.1161/01.RES.0000205766.97556.00.

49. Klempfner R, Kamerman T, Schwammenthal E et al. Efficacy of exercise training in symptomatic patients with hypertrophic cardiomyopathy: results of a structured exercise training program in a cardiac rehabilitation center. Eur J Prev Cardiol, 2015; 22: 13-19. doi: 10.1177/2047487313501277.

50. Dimitrow PP, Cotrim C, Cheng TO. The importance of upright posture in exercise testing and training for patients with hypertrophic cardiomyopathy. Eur J Prev Cardiol, 2015; 22: 354-355. doi: 10.1177/2047487314520914.

51. Pelliccia A, Fagard R, Bjørnstad HH et al. Recommendations for competitive sports participation in athletes with cardiovascular disease: a consensus document from the Study Group of Sports Cardiology of the Working Group of Cardiac Rehabilitation and Exercise Physiology and the Working Group of Myocardial and Pericardial Diseases of the European Society of Cardiology. Eur Heart J, 2005; 26: 1422-1445. doi: 10.1093/eurheartj/ehi325.

52. Pelliccia A, Zipes DP, Maron BJ. Bethesda Conference \#36 and the European Society of Cardiology Consensus Recommendations revisited a comparison of U.S. and European criteria for eligibility and disqualification of competitive athletes with cardiovascular abnormalities. J Am Coll Cardiol, 2008; 52: 1990-1996. doi: 10.1016/j.jacc.2008.08.055.

53. Światowiec A, Król W, Kuch M et al. Analysis of 12-lead electrocardiogram in top competitive professional athletes in the light of recent guidelines. Kardiol Pol, 2009; 67: 1095-1102.

54. Ho CY, Sweitzer NK, McDonough B et al. Assessment of diastolic function with Doppler tissue imaging to predict genotype in preclinical hypertrophic cardiomyopathy. Circulation, 2002; 105: 2992-2997. doi: 10.1161/01.CIR.0000019070.70491.6D.

55. Caselli S, Di Paolo FM, Pisicchio C et al. Patterns of left ventricular diastolic function in Olympic athletes. J Am Soc Echocardiogr, 2015; 28: 236-244. doi: 10.1016/j.echo.2014.09.013.

56. D’Ascenzi F, Pelliccia A, Natali BM et al. Increased left atrial size is associated with reduced atrial stiffness and preserved reservoir function in athlete's heart. Int J Cardiovasc Imaging, 2015;31: 699-705. doi: 10.1007/s10554-015-0600-7.

57. Nagueh SF, Appleton CP, Gillebert TC et al. Recommendations for the evaluation of left ventricular diastolic function by echocardiography. J Am Soc Echocardiogr, 2009; 22: 107-133. doi: 10.1016/j.echo.2008.11.023.

58. Teare D. Asymmetrical hypertrophy of the heart in young adults. Br Heart J. 1958; 20: 1-8.

59. Hollman A, Goodwin JF, Teare D, Renwick JW. A family with obstructive cardiomyopathy (asymmetrical hypertropthy). Br Heart J, 1960; 22: 449-456.

60. Dimitrow PP, Chojnowska L, Rudzinski T et al. Sudden death in hypertrophic cardiomyopathy: old risk factors re-assessed in a new model of maximalized follow-up. Eur Heart J, 2010; 31: 3084-3093. doi: 10.1093/eurheartj/ehq308.

Cite this article as: Krysztofiak H, Petkow Dimitrow P. Differentiating physiology from pathology in elite athletes. Left ventricular hypertrophy versus hypertrophic cardiomyopathy. Kardiol Pol, 2016; 74: 705-716. 10.5603/KP.a2016.0084. 